\title{
K-carrageenan/chitosan nanolayered coating for controlled release of a model bioactive compound
}

\author{
Ana C. Pinheiro a , Ana I. Bourbon ${ }^{a}$, Mafalda A.C. Quintas ${ }^{\text {a,b }}$, Manuel A. Coimbra ${ }^{c}$, António A. Vicente ${ }^{\text {a,* }}$ \\ ${ }^{a}$ IBB - Institute for Biotechnology and Bioengineering, Centre of Biological Engineering, University of Minho, Campus de Gualtar, 4710-057 Braga, Portugal \\ ${ }^{\text {b } C B Q F}$ / Escola Superior de Biotecnologia, Rua Dr Antonio Bernardino de Almeida, 4200-072 Porto, Portugal \\ c QOPNA, Department of Chemistry, University of Aveiro, 3810-193 Aveiro, Portugal
}

\section{A R T I C L E I N F O}

\section{Article history:}

Received 3 October 2011

Accepted 13 June 2012

\section{Keywords:}

Nanolayers

Polyelectrolyte multilayers

Nanocoatings

Layer-by-layer

Methylene Blue

Fick's diffusion

\begin{abstract}
A B S T R A C T
Multilayer nanocoatings composed of $\kappa$-carrageenan, a sulphated anionic polysaccharide, and chitosan, a cationic polysaccharide, were produced by layer-by-layer deposition. The model cationic compound Methylene Blue (MB) was incorporated in different positions of the nanolayered coating and its loading and release behavior was evaluated. UV-VIS spectroscopy and quartz crystal microbalance analysis showed that the amount of MB loaded increased with the distance from the first layer, suggesting that the MB was able to diffuse into the K-carrageenan/chitosan nanolayered coating and not only adhered to the surface of the layer immediately below it. For most of the tested conditions, the MB release from the $\kappa$-carrageenan/chitosan nanolayered coatings was successfully described by the linear superimposition model, which allowed concluding that $\mathrm{MB}$ transport is due to both concentration gradient and the polymer relaxation of the nanolayers. However, depending on temperature and $\mathrm{pH}$ of the medium and on the position of MB incorporated on the nanolayered coatings, different mechanisms prevail.

Industrial relevance: The development of novel edible coatings with improved functionality and performance for e.g. fresh and minimally processed fruits is one of the challenges of the post-harvest industry. This work contributes to the understanding of the loading and release phenomena involved in structures at the nanoscale, which is useful for the development of bioactive compounds release systems for application in food industry. Moreover, the $\kappa$-carrageenan/chitosan nanolayered coatings represent a promising platform from which the controlled release of different bioactive compounds may be explored.
\end{abstract}

(c) 2012 Elsevier Ltd. All rights reserved.

\section{Introduction}

Over the past years, there has been an increasing interest in the field of bioactive compounds delivery, resulting in new controlled release systems that provide continuous liberation of the functional compounds. However, particularly in the food industry, the development of versatile and highly responsive release systems still constitutes a challenge. Nanolayered coatings have promising applications in a wide range of areas including pharmaceutical, biomedical and food packaging. Particularly, when applied on food products, they can be used as a strategy for shelf-life extension. However, there is a need to create systems of multilayers that combine both biofunctionality and edibility. Conventional layer-by-layer (LbL) assembly is based on the alternating deposition of oppositely charged polyelectrolytes and can be used to produce multilayers with thickness control on the nanometer scale (Decher \& Schlenoff, 2003). In general, the resulting polyelectrolyte multilayer films/coatings are formed by interpenetrated polyelectrolyte chains and not by a strictly stratified structure (Decher, 1997).

\footnotetext{
* Corresponding author. Tel.: +351253 604419; fax: +351253678986.

E-mail address: avicente@deb.uminho.pt (A.A. Vicente).
}

More recently, LbL nanolayered film assemblies have been investigated as drug release systems (Chung \& Rubner, 2002; Jiang \& Li, 2009; Zhong, Whittington, Zhang, \& Haynie, 2007) due to the possibility to control the drug release through manipulating the film/coating properties and to incorporate a wide range of functional biomolecules without substantial loss of their biological functions (Wang et al., 2007). The functional LbL multilayers can be obtained by chemical grafting of polyelectrolytes by functional moieties (Kaschak et al., 1999), alternate deposition of polyelectrolytes and functional molecules (Rousseau, Van der Auweraer, \& De Schryver, 2000) or by post diffusion of the functional molecules into the multilayers (Jiang \& Li, 2009; Zhong et al., 2007). A diversity of multilayered films has been developed to control bioactive compounds release by deploying different triggers such as $\mathrm{pH}$, ionic strength, temperature and enzymes (Quinn \& Caruso, 2004; Serizawa, Yamaguchi, \& Akashi, 2002; Wood, Boedicker, Lynn, \& Hammond, 2005).

Chitosan, a cationic polysaccharide obtained by deacetylation of chitin, is an excellent edible film component due to its good oxygen barrier properties and due to its intrinsic antimicrobial activity (Begin \& Van Calsteren, 1999) and antioxidant activity (Xia, Liu, Zhang, \& Chen, 2011). Moreover, properties such as biodegradability, non-toxicity and biocompatibility make chitosan suitable for application in pharmaceutical and food 
products (Dutta, Tripathi, Mehrotra, \& Dutta, 2009; Illum, JabbalGill, Hinchcliffe, Fisher, \& Davis, 2001).K-carrageenan, a sulphated anionic polysaccharide extracted from certain red seaweeds, is extensively used in food industry as gelling and stabilizing agent and has been reported as having excellent film forming properties (Park, Lee, Jung, \& Park, 2001). A nanolayered coating composed of $\kappa$-carrageenan and chitosan was previously developed and it has proved to exhibit good gas barrier properties (Pinheiro et al., 2012). Therefore, this system offers great potential to be used to coat food systems such as fruit, vegetables or cheese and to act as a support for the incorporation of bioactive compounds. Methylene Blue (MB), a monovalent cation of $374 \mathrm{Da}$, is highly soluble in water and is a suitable model for loading and release experiments of small molecules due to its large absorption peak in the visible range. MB has been used to investigate multilayer surface properties due to its ability to bind to available negatively charged functional groups (Chung \& Rubner, 2002). Moreover, the loading of dye molecules, such as MB, to preformed layers offers the possibility to evaluate the extent of electrostatic interactions between oppositely charged polyelectrolytes in the multilayer films/coatings (Tedeschi, Caruso, Mohwald, \& Kirstein, 2000; Yoo, Shiratori, \& Rubner, 1998).

The release of bioactive compounds from polymeric matrices may occur due to mechanisms of Fick's diffusion, polymer matrix swelling, polymer erosion, and degradation (Faisant, Siepmann, \& Benoit, 2002; Jain, 2000) and a different mechanism may prevail, depending on the system and environmental conditions. In recent years, several works concerning the release of functional compounds from conventional edible films have been done (Del Nobile, Conte, Incoronato, \& Panza, 2008; Flores, Conte, Campos, Gerschenson, \& Del Nobile, 2007). Comparatively, few works can be found on release mechanisms involved at the nano-scale. Literature suggests that in the case of nanolayered coatings, the release behavior depends on the permeability and on the disassembly or erosion of the multilayer structure and on other experimental variables (Jiang \& Li, 2009). Understanding the release mechanisms involved, by recurring to mathematical modeling, is very important for food products development and the potential applications thereof.

In this research, multilayer nanocoatings were prepared by LbL deposition using $\kappa$-carrageenan and chitosan onto a substrate (polyethylene terephthalate - PET). The model compound MB was incorporated at different positions of the nanolayered coating and its loading and release behavior was evaluated. Diffusion of MB into liquid medium was studied at different isothermal conditions and mathematical models were used to understand the transport mechanisms.

\section{Materials and methods}

\subsection{Experimental procedures}

\subsubsection{Preparation of nanolayered coating}

The polyelectrolyte solution of $\kappa$-carrageenan was prepared dissolving $0.2 \%(\mathrm{w} / \mathrm{v})$ of $\kappa$-carrageenan (GENUGEL carrageenan type WR-78, CPKelco, Denmark) in distilled water and the $\mathrm{pH}$ of the solution was adjusted to 7.0 with a solution of $1 \mathrm{M}$ sodium hydroxide (Riedel-de Haën, Germany). The polyelectrolyte solution of chitosan was prepared dissolving $0.2 \%(\mathrm{w} / \mathrm{v})$ of chitosan $\left(90 \%\right.$ deacetylation, $\mathrm{MW}=3.4 \times 10^{5}$, Aqua Premier Co. Ltd, Thailand) in a $1.0 \%(\mathrm{v} / \mathrm{v})$ lactic acid solution and the $\mathrm{pH}$ of the solution was adjusted to 3.0 with a solution of $1 \mathrm{M}$ sodium hydroxide (Riedel-de Haën, Germany). MB solution was prepared dissolving $0.3 \%(\mathrm{w} / \mathrm{v})$ of MB (Riedel-de Haën, Germany) in 50\% (v/v) $0.01 \mathrm{M}$ Phosphate Buffer Solution (PBS) and ultra-pure water, and the $\mathrm{pH}$ was adjusted to 7.0 with a solution of $1 \mathrm{M}$ hydrochloric acid (Merck, Darmstadt, Germany).

The $\mathrm{pH}$ values chosen for k-carrageenan, chitosan and MB solutions are the pH's at which these polyelectrolytes exhibit the highest $\zeta$-potential values (positive or negative), thus maximizing the electrostatic interactions between these polyelectrolytes.

The nanolayered coating was composed by an aminolyzed/ charged PET (A/C PET) (Canson, France) sustaining layer adsorbed with a polysaccharide multilayer constituted of 5 layers (three $\kappa$ carrageenan and two chitosan layers). The MB was incorporated in the $\kappa$-carrageenan/chitosan nanolayered coating, forming the second

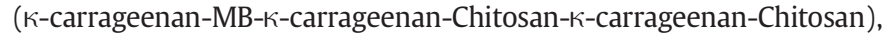

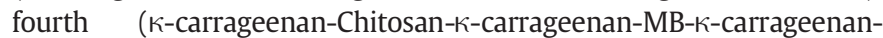

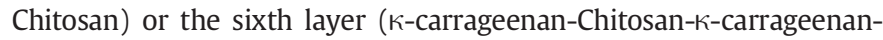
Chitosan-к-carrageenan-MB), thus replacing the corresponding chitosan layer in those positions (Fig. 1).

In order to prepare the nanolayered coating, the PET pieces were aminolyzed (Carneiro-da-Cunha et al., 2010) and were firstly dipped into the $\kappa$-carrageenan solution for $15 \mathrm{~min}$ and subsequently rinsed with distilled water at $\mathrm{pH}$ 7. The samples were dried with a flow of nitrogen. The procedure was repeated, this time using chitosan (or MB) as the polyelectrolyte and rinsed with distilled water at $\mathrm{pH} 3.0$ (or 7.0). The dipping/ washing/drying process was repeated until the deposition of the desirable layers conformation was achieved. The nanolayered coatings were then maintained at $20{ }^{\circ} \mathrm{C}$ and $50 \%$ relative humidity ( $\mathrm{RH}$ ) before analysis.

\subsubsection{MB loading}

The loading of MB on each position of the multilayer structure was monitored by UV-VIS spectroscopy (Jasco 560, Germany), in the range $190-800 \mathrm{~nm}$. The maximum MB absorption peak in the nanolayered coating was $570 \mathrm{~nm}$. Three replicates were performed.

Real time deposition of MB on the coated electrode was recorded by Quartz Crystal Microbalance (QCM 200 purchased from Stanford Research Systems, SRS, USA), equipped with AT-cut quartz crystals ( $5 \mathrm{MHz}$ ) with optically flat polished titanium/gold electrodes on contact and liquid sides. Three replicates were performed.

The deposition of the layers and their nano dimensions were also confirmed by scanning electron microscopy (SEM) (Nova NanoSEM 200, Netherlands) with an accelerating voltage from 10 to $15 \mathrm{kV}$. Before analyses, all samples were mounted on aluminum stubs using carbon adhesive tape and sputter-coated with gold (thickness of about $10 \mathrm{~nm}$ ). The SEM images allowed the direct measurement of the layers thicknesses using the equipment's image analysis software (XT microscope control software).

\subsection{3. $M B$ release}

The release of MB from the multilayer coating on PET was evaluated by incubating the loaded coatings in $40 \mathrm{~mL}$ of $0.01 \mathrm{M}$ PBS of a certain $\mathrm{pH}$ ( 2.0 or 7.0 ) and temperature ( 4 or $37^{\circ} \mathrm{C}$ ). The release medium was continuously stirred at $60 \mathrm{rpm}$. At preset intervals, $0.25 \mathrm{ml}$ supernatant was taken and $0.25 \mathrm{ml}$ of fresh PBS was added to keep the volume of the release medium constant. The sample solutions of MB were analyzed using UV-VIS spectroscopy (Jasco 560, Germany) by measuring their absorbance at $600 \mathrm{~nm}$ - the maximum absorption peak of MB in solution. Three replicates were conducted for each condition.

\subsection{Statistical procedures}

The equations mentioned along the text were fitted to data by nonlinear regression analysis, using a package of STATISTICA ${ }^{\text {TM }} 7.0$ (Statsoft. Inc, USA). The Levenberg-Marquadt algorithm for the least squares function minimization was used.

The quality of the regressions was evaluated on the basis of the determination coefficient, $R^{2}$, the squared root mean square error, RMSE (i.e., the square root of the sum of the squared residues (SSE) divided by the regression degrees of freedom) and residuals visual inspection for randomness and normality. $R^{2}$ and SSE were obtained directly from the software. 


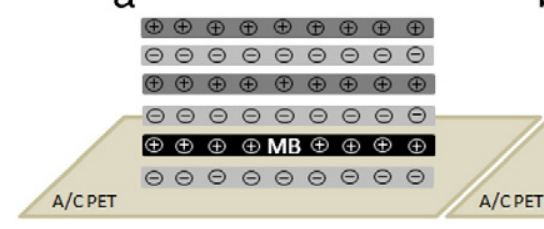

$\oplus \oplus \oplus \mathrm{MB}$

$\Theta \ominus \ominus \boldsymbol{x}$-carrageenan

$\oplus \oplus \oplus$ Chitosan b

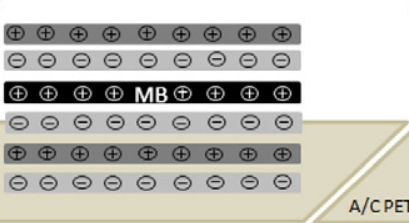

$\mathrm{C}$

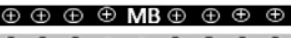

$\ominus \ominus \ominus \ominus \ominus \ominus \ominus \ominus \ominus$ $\oplus \oplus \oplus \oplus \oplus \oplus \oplus \oplus \oplus$ $\ominus \ominus \ominus \ominus \ominus \ominus \ominus \ominus \ominus$ $\oplus \oplus \oplus \oplus \oplus \oplus \oplus \oplus$

Fig. 1. Schematic representation of the MB loading in the different positions of the א-carrageenan/chitosan nanolayered: 2 nd layer (a), 4th layer (b) and 6th layer (c).

The precision of the estimated parameters was evaluated by the Standardized Halved Width (SHW \%), which was defined as the ratio between the 95\% Standard Error (obtained from the software) and the value of the estimate.

\section{Results and discussion}

\subsection{MB loading}

Nanolayered film/coating architecture is important for bioactive compound loading. The MB loading at different positions of the $\mathrm{k}$ carrageenan/chitosan multilayered coating was evaluated by UV-VIS spectroscopy (Fig. 2). For all cases, the maximum absorbance was observed at $570 \mathrm{~nm}$, which corresponds to a characteristic absorption peak of $\mathrm{MB}$, thus substantiating the incorporation of this compound in the $\kappa$-carrageenan/chitosan nanolayered coatings.

It can be seen that the MB absorbance increased with the distance from the first layer. This can be due to the fact that full establishment of the layer organization can occur only after deposition of a minimum number of layers, depending on the system (Yoo et al., 1998). Literature proposes that before this minimum number of layers is achieved, interpenetration between layers can occur (Decher, 1997). This phenomenon may lead to uneven deposition in the first layers of the nano-structure and thus less charged chain segments for adhesion of $\mathrm{MB}$ are available (Yoo et al., 1998; Decher, 1997). Also, it is possible that the MB adsorption is not confined to the outermost surface but MB was able to diffuse into the $\kappa$-carrageenan/chitosan nanolayered coating. If that was the case, as the multilayer coating was built, coating's total thickness increased and consequently more MB was loaded (Chung \& Rubner, 2002).

The interpenetration and diffusion phenomena can also be inferred by the results of real-time adsorption of MB on $\kappa$-carrageenan/chitosan pre-coated gold electrode surface, which were monitored using a quartz crystal microbalance (QCM). The mass changes as a function of time for

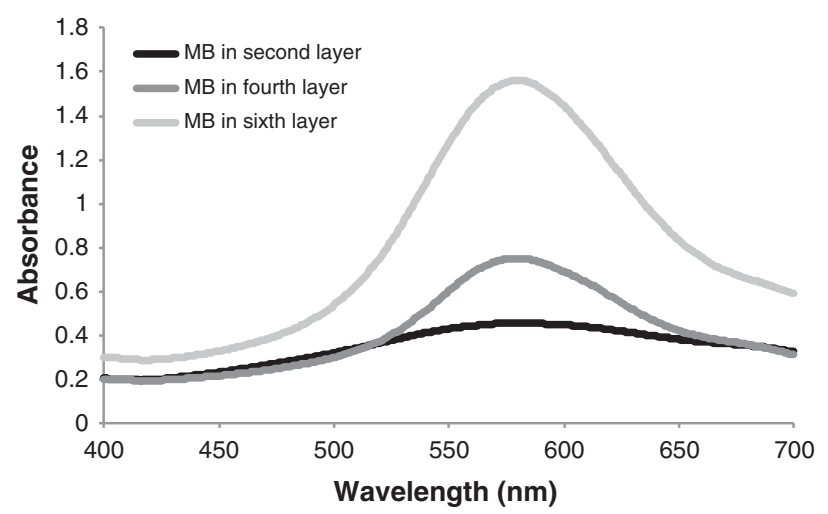

Fig. 2. UV-VIs absorption spectra of the $k$-carrageenan/chitosan coatings as a function of the MB layer position in the multilayer structure. the deposition of MB constituting the second, fourth or sixth layer of the multilayer structure are shown in Fig. 3.

It was found that MB loaded mass increased with the incubation time, exhibiting a very fast initial adsorption phase followed by a slower phase upon approaching a steady state.

The initial fast adsorption phase shows an increase of total MB loaded with the number of layer, with the MB loading capacity in the sixth layer of the multilayer structure being about ten times that in the second layer. Since the incorporation of MB in the $\kappa$-carrageenan/chitosan nanolayered coating is mainly driven by the electrostatic interaction between $M B$ and $\kappa$-carrageenan molecules and the amount of MB adsorbed depends mainly on the number of anionic groups of $\kappa$-carrageenan not forming contact ion pairs with cationic groups of chitosan (i.e., on the number of anionic groups available for further interactions) (Soedjak, 1994), the increase of MB loading with layer number supports the existence of high interpenetration between layers in the innermost layers and of a more homogenous surface as the layers are consecutively deposed.

As for the slower phase of MB loading, it can be a reflection of MB diffusion into the nanolayered coating. The increase of the slope of the curve with layer number observed in Fig. 3 supports the existence of such phenomenon and that it is proportional to coating's thickness.

From these results, one can conclude that it is possible to control the amount of bioactive compound adsorbed by simply controlling its position in the multilayer structure.

The scanning electron microscopy (SEM) images of the $\kappa$ carrageenan/chitosan coating allowed confirming the nano-scale of the obtained layers. Fig. 4 shows the SEM image of the coating with $\mathrm{MB}$ in the 6th layer as an example. The total thickness of the $\kappa-$ carrageenan/chitosan layers was $234.9 \mathrm{~nm}$ (Fig. 4a). Also, a detailed image (Fig. 4b) shows the structure of the layers, revealing that kcarrageenan/chitosan coating is formed by interpenetrated layers and not by strictly stratified layers.

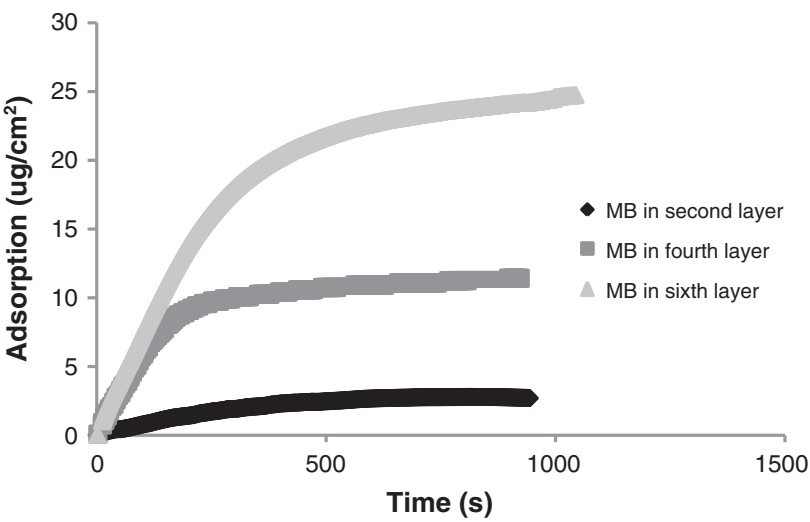

Fig. 3. Real time monitoring of the deposition of MB forming the second, fourth and sixth layer of the K-carrageenan/chitosan multilayered coatings using a Quartz crystal microbalance (QCM). 


\section{2. $M B$ release}

Experimentally, all the release profiles were characterized by three different parts: an initial burst phase, continuous release phase and a stagnant phase. These results can be interpreted on the basis of mass transport theory: Pure diffusion, polymer matrix swelling, polymer erosion and degradation are mechanisms that lead to compound release from polymeric devices (Faisant et al., 2002; Jain, 2000; Polakovic, Görner, Gref, \& Dellacherie, 1999). Depending on both the system (polymer/active compound) and environmental conditions, a different mechanism may prevail. These mechanisms may be generally classified as of three different types: ideal Fickian diffusion (Brownian transport); Case II transport (polymer relaxation driven) and anomalous behavior (ranging from Fickian to Case II transport). At the nano-scale, few works in literature address compound release mechanisms from layered coatings. Literature suggests that, depending on nanolayered coatings composition and on the released compound, release may follow a Fickian or an anomalous transport behavior (Wang et al., 2007).

The Linear Superimposition Model accounts for both Fickian and case II transport effects on the observed anomalous behavior in hydrophilic matrices (Berens \& Hopfenberg, 1978) and is then a suitable approach to investigate transport mechanism in these systems.

This approach assumes that the observed transport of molecules within the polymer can be described by the sum of the molecules transported due to Brownian motion (Fick's transport) with the molecules transported due to polymer relaxation.

For a thin slab of polymer immersed in a sufficiently large amount of water, molecular transport due to Brownian motion can be described by the solution of Fick's second law for a plane sheet with constant boundary conditions (Crank, 1975), which can be simplified using the first term of the Taylor series (Rathore \& Kapuno, 2011):

$\frac{M_{t}}{M_{\infty, F}}=1-\frac{8}{\pi^{2}} \exp \left(-k_{F} t\right)$

where $M_{t}$ is the total mass released from the polymer at time $t, M_{\infty, F}$ is the compound release at equilibrium and $k_{F}$ is Fickian diffusion rate constant.

As for polymer relaxation, it is driven by the swelling ability of the polymer and is then related to the dissipation of stress induced by the entry of the penetrant and can be described as a distribution of relaxation times, each following a first order-type kinetic equation (Berens \& Hopfenberg, 1978).

$M_{t}=\sum_{i} M_{\infty, R i}\left[1-\exp \left(-k_{R i} t\right)\right]$

here, $\mathrm{M}_{\infty, R i}$ are the contributions of the relaxation processes for compound release and $k_{R i}$ are the relaxation $i$ th rate constants. For most cases, there is only one main polymer relaxation that influences transport and thus the above equation can be simplified using $i=1$.
Assuming the above conditions, the Linear Superimposition model for compound release from a hydrophilic polymer slab yields (Flores et al., 2007):

$$
\frac{M_{t}}{M_{\infty}}=X\left[1-\frac{8}{\pi^{2}} \exp \left(-k_{F} t\right)\right]+(1-X)\left[1-\exp \left(-k_{R} t\right)\right]
$$

where $X$ is the fraction of compound released by Fickian transport.This "general" model can then be used to describe Fickian $\left(\mathrm{M}_{\infty, F} \neq 0\right.$ and $i=0)$; anomalous $\left(\mathrm{M}_{\infty, F}\right.$ and $\left.i \neq 0\right)$ or Case II transport $\left(\mathrm{M}_{\infty, F}=0\right.$ and $i \neq 0)$.

To assess the transport mechanism at the nano-scale and investigate the phenomena observed at this scale, release experimental results were analyzed by fitting Eqs. (1) and (2) to the obtained data. The effects of temperature, $\mathrm{pH}$ and layer position on MB release were evaluated.

\subsubsection{Assessment of Fick's transport in nanolayered coatings}

In all release experiments carried under different conditions (temperature 4 and $37^{\circ} \mathrm{C}$; pH 2 and 7) and from the different layers (2nd, 4 th and 6th), results from fitting Eq. (1) lead to a poor description of the data (see examples on Figs. 5 and 6). This indicates that transport mechanism for the tested nanolayered coatings cannot be described by only Brownian motion of MB molecules in the polymeric matrix, i.e. it does not follow Fick's behavior.

\subsubsection{Anomalous transport in nanolayered coatings}

As for fitting Eq. (2) - i.e. assuming anomalous behavior with one main relaxation - to the experimental data, a successful description was achieved for most of the tested conditions. However, different results can be observed depending on the position of MB incorporated on the nanolayered coating or the $\mathrm{pH}$ and temperature of the medium.

\section{3. $M B$ release from the outermost layer}

Fig. 5a shows an example of the adequate description of the LSM (Eq. (2)) of release of MB from the 6th layer and Table 1 presents the regression analysis results. It can be observed that this model adequately describes the experimental data with good regression quality and parameters are estimated with good precision (maximum SHW\% was $57 \%$ ).

These results show that in the outermost layer, the mechanism of diffusion is governed by both Fickian and Case II transport, with only one main relaxation of the polymeric coating. The fact that polymer relaxation has an influence on $\mathrm{MB}$ release supports the existence of interpenetration between layers even between $\mathrm{MB}$ in the outermost and $\kappa$-carrageenan on the 5th layers and/or diffusion of MB into the inner layers of the nano-device.

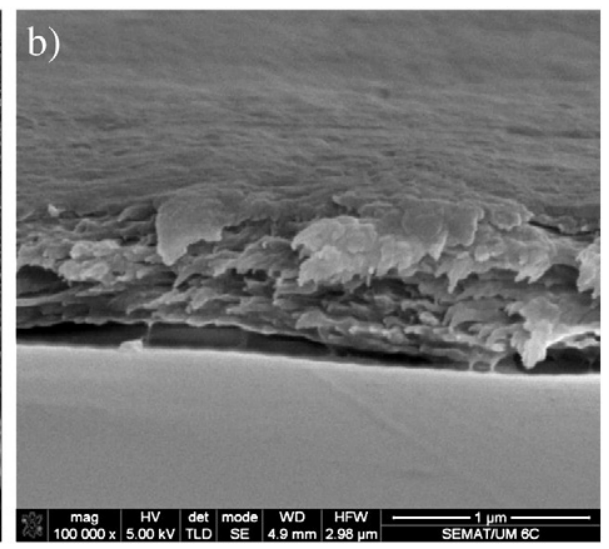

Fig. 4. SEM images of the K-carrageenan/chitosan coating with MB in the 6th layer. Thickness of the layers (a). Detail of the structure of the layers (b). 

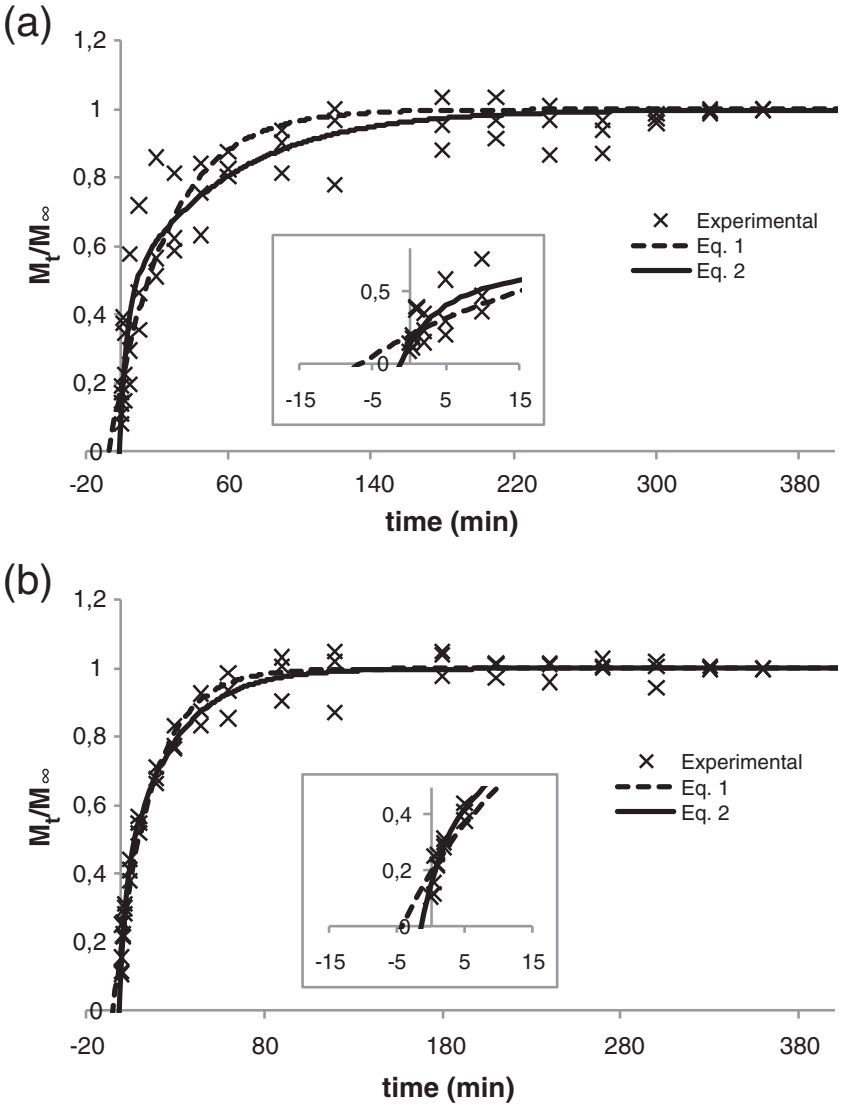

Fig. 5. Example of Fick's (Eq. 1) and LSM (Eq. 2) description of Methylene Blue release at $37^{\circ} \mathrm{C}$ from: (a) the 6 th layer at $\mathrm{pH}=7$ and (b) the 4 th layer at $\mathrm{pH}=2$. Insets show the detail of the model fitting to the initial experimental data.

The knowledge of the effects of the environmental conditions (such as temperature and $\mathrm{pH}$ ) on the quantity of mass released and on the rate of release can be of greatest importance for the application of these systems on food products. Furthermore, once applied on a food system, the release of the bioactive compound can occur after or before ingestion. Therefore, the release behavior of $\mathrm{MB}$ from $\kappa$-carrageenan/chitosan nanolayered coatings was evaluated at 37 and $4{ }^{\circ} \mathrm{C}$, which are the temperatures within the human body and the standard refrigeration temperature of a food product, respectively, and at a $\mathrm{pH}$ of 2.0 and 7.0, which are the pHs corresponding to the conditions within a human gastrointestinal tract (particularly in the stomach and in the small intestine, respectively). As

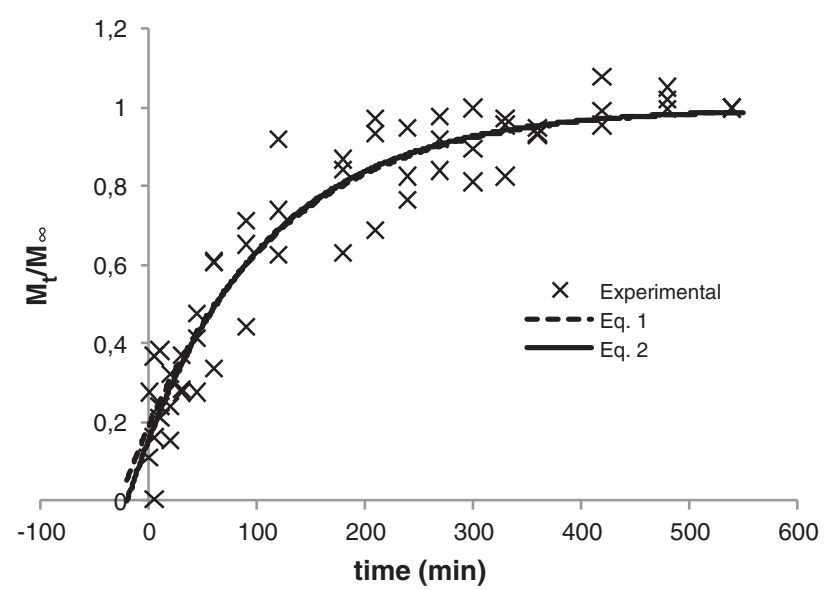

Fig. 6. Fick's (Eq. (1)) and LSM (Eq. (2)) description of Methylene Blue release at pH 7. Example is for the 4 th layer at $4{ }^{\circ} \mathrm{C}$.
Table 1

Results of fitting the Linear Superimposition Model (LSM) (Eq. (2)) to experimental data of the MB release. Evaluation of the quality of the regression on the basis of RMSE and $R^{2}$. Estimates precision is evaluated using the SHW\% (in parenthesis).

\begin{tabular}{|c|c|c|c|c|c|c|c|}
\hline $\begin{array}{l}\text { Layer } \\
\text { position }\end{array}$ & $\mathrm{pH}$ & $\begin{array}{l}\text { Temp } \\
\left({ }^{\circ} \mathrm{C}\right)\end{array}$ & RMSE & $R^{2}$ & $X$ & $k_{F}\left(\min ^{-1}\right)$ & $k_{R}\left(\min ^{-1}\right)$ \\
\hline \multirow[t]{4}{*}{6} & \multirow[t]{2}{*}{7} & 37 & $3.82 \times 10^{-2}$ & 0.9854 & $\begin{array}{l}0.775 \\
(15 \%)\end{array}$ & $\begin{array}{l}3.58 \times 10^{-2} \\
(18 \%)\end{array}$ & $\begin{array}{l}2.81 \times 10^{-1} \\
(49 \%)\end{array}$ \\
\hline & & 4 & $7.96 \times 10^{-2}$ & 0.9461 & $\begin{array}{l}0.661 \\
(44 \%)\end{array}$ & $\begin{array}{l}1.27 \times 10^{-2} \\
(41 \%)\end{array}$ & $\begin{array}{l}3.51 \times 10^{-2} \\
(57 \%)\end{array}$ \\
\hline & \multirow[t]{2}{*}{2} & 37 & $7.52 \times 10^{-2}$ & 0.9378 & $\begin{array}{l}0.464 \\
(40 \%)\end{array}$ & $\begin{array}{l}2.93 \times 10^{-2} \\
(55 \%)\end{array}$ & $\begin{array}{l}3.19 \times 10^{-1} \\
(40 \%)\end{array}$ \\
\hline & & 4 & $4.05 \times 10^{-2}$ & 0.9800 & $\begin{array}{l}0.421 \\
(45 \%)\end{array}$ & $\begin{array}{l}2.25 \times 10^{-2} \\
(41 \%)\end{array}$ & $\begin{array}{l}8.16 \times 10^{-2} \\
(20 \%)\end{array}$ \\
\hline \multirow[t]{2}{*}{2} & \multirow[t]{2}{*}{2} & 37 & $6.60 \times 10^{-2}$ & 0.9635 & $\begin{array}{l}0.124 \\
(258 \%)\end{array}$ & $\begin{array}{l}7.20 \times 10^{-3} \\
(228 \%)\end{array}$ & $\begin{array}{l}1.30 \times 10^{-2} \\
(26 \%)\end{array}$ \\
\hline & & 4 & $9.12 \times 10^{-2}$ & 0.9246 & $\begin{array}{l}0.734 \\
(35 \%)\end{array}$ & $\begin{array}{l}5.70 \times 10^{-3} \\
(33 \%)\end{array}$ & $\begin{array}{l}3.56 \times 10^{-2} \\
(88 \%)\end{array}$ \\
\hline \multirow[t]{2}{*}{4} & \multirow[t]{2}{*}{2} & 37 & $9.52 \times 10^{-2}$ & 0.9068 & $\begin{array}{l}0.656 \\
(29 \%)\end{array}$ & $\begin{array}{l}1.67 \times 10^{-2} \\
(44.24 \%)\end{array}$ & $\begin{array}{l}2.21 \times 10^{-1} \\
(76 \%)\end{array}$ \\
\hline & & 4 & $1.01 \times 10^{-1}$ & 0.8994 & $\begin{array}{l}0.650 \\
(31 \%)\end{array}$ & $\begin{array}{l}5.40 \times 10^{-3} \\
(37 \%)\end{array}$ & $\begin{array}{l}5.77 \times 10^{-2} \\
(83 \%)\end{array}$ \\
\hline
\end{tabular}

can be evaluated from Table 1, MB release from the 6th layer was influenced by both temperature and $\mathrm{pH}$. Although these effects were not significant $(p>0.05)$ they provide a good insight on the behavior of the polymeric nanolayered coating at the different environmental conditions tested.

Fick's diffusion contribution to the total release from the nanolayered coating can be evaluated by the estimate of $X$ (which is defined as $\frac{M_{\infty, F}}{M_{t}}$ ). It can be observed that for neutral $\mathrm{pH}(7), X$ is higher than 0.5 , indicating that Fick's is the main release mechanism in this condition. As for the $\mathrm{MB}$ release in $\mathrm{pH}=2$ relaxation is the governing phenomenon $(X<0.5)$. At $\mathrm{pH} 2.0$, the electrostatic interaction between $\mathrm{K}$-carrageenan and $\mathrm{MB}$ is very low, since the pKa value of the anionic sulfate groups on $\mathrm{k}$ carrageenan is around $\mathrm{pH} 2$ and at this $\mathrm{pH}$ the sulfate groups on the $\kappa$ carrageenan molecules are half protonated and consequently partially uncharged (Gu, Decker, \& McClements, 2005). The decrease of electrochemical interaction with chitosan or MB facilitates the loosening of the polymer chain network and promotes release due to polymer relaxation.

In both studied $\mathrm{pH}$ conditions, the amount of $\mathrm{MB}$ released through Fick's transport (evaluated by $X$ estimate) increased with process temperature - this is of course not unexpected - a higher internal energy of a system will promote increased molecular vibration and movement, i.e. Brownian motion; and increased solubility of diffusing molecules (Myint, Daud, Mohamad, \& Kadhum, 1996; Vojdani \& Torres, 1990). Similarly, the Fickian and relaxation rate constants $\left(k_{F}\right.$ and $\left.k_{R}\right)$ increased with temperature.

The relaxation rate constant $\left(k_{R}\right)$, decreased with increasing $\mathrm{pH}$ for both tested temperatures. This supports the previously discussed effect of acidic $\mathrm{pH}$ on promoting transport through relaxation of the polymeric network.

As for $k_{F}$, the Fickian rate constant, the effect of $\mathrm{pH}$ was different at the two tested temperatures. While at $37^{\circ} \mathrm{C} k_{F}$ increased with $\mathrm{pH}$, thus reflecting Fick's behavior for the tightly packed polymer network at neutral $\mathrm{pH}$, at $4{ }^{\circ} \mathrm{C}$ this parameter showed an unexpected inverse behavior $-k_{F}$ was higher at $\mathrm{pH}=2$. This observation does not mean that Fick's diffusion was higher than polymer relaxation under this condition, but it shows that for a system with low internal energy (i.e. low temperature) and for a tightly packed polymer network $(\mathrm{pH}=7)$ the molecular mobility can be decreased to a point where even Fick's diffusion is limited.

\section{4. $M B$ release from the 2 nd and 4 th layers}

MB release in the inner layers (2nd and 4th) presented a different behavior than the one previously discussed. 
Under acidic conditions ( $\mathrm{pH}=2$ ), the transport of $\mathrm{MB}$ can be described by the LSM (Eq. (2)), as shown on Fig. 5b. Results of the regression analysis (Table 1) show that although the model describes the experimental data, parameters are estimated with low precision (maximum SHW\% was 258\%), hindering any conclusion on the effect of environmental conditions on the diffusion kinetics.

For the inner layers, at neutral $\mathrm{pH}$, both Eqs. (1) and (2) were not able to describe the experimental data (see Fig. 6). This is related with the fact that, as discussed above, $\mathrm{k}$-carrageenan is negatively charged at $\mathrm{pH} 7$, while chitosan and $\mathrm{MB}$ are positively charged, leading to electrochemical interaction. This stronger interaction affects the transport mechanism, which cannot be described by either Fickian or anomalous behavior.

These results show that the MB position on the nanolayered coating influences its release. As expected, at the same release conditions, the $\mathrm{MB}$ in the sixth layer enabled a more extensive and faster release of this compound, when compared with the MB in the fourth or second layers. Therefore, depending on the application, the quantity of the mass loaded and consequently released and the rate of release can be controlled by simple altering the position of the compound of interest within the nanolayered structure.

\subsubsection{Other mechanisms of transport in nanolayered coatings}

One should consider that other factors that are not accounted in the Linear Superimposition Model (such as the disassembly or erosion of the multilayer structure) may be involved on MB release (Jiang \& Li, 2009). As an initial approach to this question, the diffusion and relaxation rate constants estimated at the different nano-layers can be compared. For $\mathrm{pH}=2$, the condition where $k_{R}$ and $k_{F}$ could be estimated in all the studied layers, it was observed that the resistance to transport was higher in the inner layers. This may be an indication that the disassembly or the erosion of the multilayer structure was not a predominant factor in this case.

From these results, one can conclude that the main physical mechanism of the MB transport in nanolayered $\kappa$-carrageenan/chitosan coatings is anomalous transport, with one main polymer relaxation. Similar results were obtained by other authors that observed that the release of azoalbumin is a complex combination of pure diffusion and Case II transport (Wang et al., 2007).

\section{Conclusions}

The results obtained in this work provide useful information on the morphology and architecture of the nanolayered coatings. UV-VIS spectroscopy and quartz crystal microbalance analysis demonstrated that the amount of MB loaded increased with the distance from the first layer, suggesting layer interpenetration of the inner layers and that MB was able to diffuse into the $\kappa$-carrageenan/chitosan nanolayered coating.

The results of fitting the Linear Superimposition Model to the experimental data of $\mathrm{MB}$ release suggest an anomalous behavior, with one main polymer relaxation. The effects of layer position, temperature and $\mathrm{pH}$ on $\mathrm{MB}$ release were evaluated and different results were observed depending on the position of MB incorporated on the nanolayered coating or the $\mathrm{pH}$ and temperature of the medium.

This work allows clarifying the release mechanisms involved at nano-scale, which is of the utmost importance for the application of nanolayered systems in food products, as a strategy for shelf-life extension.

\section{Acknowledgments}

The authors Ana C. Pinheiro, Ana I. Bourbon and Mafalda A.C. Quintas are recipient of fellowships from the Fundação para a Ciência e Tecnologia (FCT, Portugal) through grants SFRH/BD/48120/2008, SFRH/BD/73178/ 2010 and SFRH/BPD/41715/2007, respectively. This work was supported by Fundação para a Ciência e a Tecnologia through project NANO/NTec-
SQA/0033/2007. The authors also acknowledge Dr. Edith Ariza from SEMAT/UM by the support in SEM analysis.

\section{References}

Begin, A., \& Van Calsteren, M. R. (1999). Antimicrobial films produced from chitosan. International Journal of Biological Macromolecules, 26(1), 63-67.

Berens, A. R., \& Hopfenberg, H. B. (1978). Diffusion and relaxation in glassy polymer powders. 2. Separation of diffusion and relaxation parameters. Polymer, 19(5), 489-496.

Carneiro-da-Cunha, M. G., Cerqueira, M. A., Souza, B. W. S., Carvalho, S., Quintas, M. A. C. Teixeira, J. A., et al. (2010). Physical and thermal properties of a chitosan/alginate nanolayered PET film. Carbohydrate Polymers, 82(1), 153-159.

Chung, A. J. \& Rubner, M. F. (2002). Methods of loading and releasing low molecular weight cationic molecules in weak polyelectrolyte multilayer films. Langmuir, 18(4), 1176-1183.

Crank, J. (1975). The mathematics of diffusion (2 ed.). Oxford: Clarendon Press.

Decher, G. (1997). Fuzzy nanoassemblies: Toward layered polymeric multicomposites. Science, 277(5330), 1232-1237.

Decher, G., \& Schlenoff, J. B. (2003). Polyelectrolyte Multilayers, an Overview. : Weinheim Wiley-VCH Verlag GmbH \& Co. KGaA.

Del Nobile, M. A., Conte, A., Incoronato, A. L., \& Panza, O. (2008). Antimicrobial efficacy and release kinetics of thymol from zein films. Journal of Food Engineering, 89(1), 57-63.

Dutta, P. K., Tripathi, S., Mehrotra, G. K., \& Dutta, J. (2009). Perspectives for chitosan based antimicrobial films in food applications. Food Chemistry, 114(4), 1173-1182.

Faisant, N., Siepmann, J., \& Benoit, J. P. (2002). PLGA-based microparticles: elucidation of mechanisms and a new, simple mathematical model quantifying drug release. European Journal of Pharmaceutical Sciences, 15(4), 355-366.

Flores, S., Conte, A., Campos, C., Gerschenson, L., \& Del Nobile, M. (2007). Mass transport properties of tapioca-based active edible films. Journal of Food Engineering, 81(3), 580-586.

Gu, Y. S., Decker, E. A., \& McClements, D. J. (2005). Influence of pH and carrageenan type on properties of beta-lactoglobulin stabilized oil-in-water emulsions. Food Hydrocolloids, 19(1), 83-91.

Illum, L., Jabbal-Gill, I., Hinchcliffe, M., Fisher, A. N., \& Davis, S. S. (2001). Chitosan as a novel nasal delivery system for vaccines. Advanced Drug Delivery Reviews, 51(1-3), 81-96.

Jain, R. A. (2000). The manufacturing techniques of various drug loaded biodegradable poly(lactide-co-glycolide) (PLGA) devices. Biomaterials, 21(23), 2475-2490.

Jiang, B. B., \& Li, B. Y. (2009). Tunable drug loading and release from polypeptide multilayer nanofilms. International Journal of Nanomedicine, 4(1), 37-53.

Kaschak, D. M., Lean, J. T., Waraksa, C. C., Saupe, G. B., Usami, H., \& Mallouk, T. E. (1999) Photoinduced energy and electron transfer reactions in lamellar polyanion/polycation thin films: toward an inorganic "leaf". Journal of the American Chemical Society, 121(14), 3435-3445.

Myint, S., Daud, W. R. W., Mohamad, A. B., \& Kadhum, A. A. H. (1996). Temperaturedependent diffusion coefficient of soluble substances during ethanol extraction of clove. Journal of the American Oil Chemists' Society, 73(5), 603-610.

Park, S. Y., Lee, B. I., Jung, S. T., \& Park, H. J. (2001). Biopolymer composite films based on [kappa]-carrageenan and chitosan. Materials Research Bulletin, 36(3-4), 511-519.

Pinheiro, A. C., Bourbon, A. I., Medeiros, B. G. S., da Silva, L. H. M., da Silva, M. C. H., Carneiroda-Cunha, M. G., et al. (2012). Interactions between k-carrageenan and chitosan in nanolayered coatings-Structural and transport properties. Carbohydrate Polymers 87(2), 1081-1090.

Polakovic, M., Görner, T., Gref, R., \& Dellacherie, E. (1999). Lidocaine loaded biodegradable nanospheres: II. Modelling of drug release. Journal of Controlled Release, 60(2-3), 169-177.

Quinn, J. F., \& Caruso, F. (2004). Facile tailoring of film morphology and release properties using layer-by-layer assembly of thermoresponsive materials. Langmuir, 20(1), 20-22.

Rathore, M. M., \& Kapuno, R. (2011). Engineering Heat Transfer (2nd ed.). London, UK: Jones \& Bartlett Learning.

Rousseau, E., Van der Auweraer, M., \& De Schryver, F. C. (2000). Steady-state and timeresolved spectroscopy of a self-assembled cyanine dye multilayer. Langmuir, 16(23), 8865-8870.

Serizawa, T., Yamaguchi, M., \& Akashi, M. (2002). Enzymatic hydrolysis of a layer-by-layer assembly prepared from chitosan and dextran sulfate. Macromolecules, 35(23), 8656-8658.

Soedjak, H. S. (1994). Colorimetric determination of carrageenans and other anionic hydrocolloids with methylene-blue. Analytical Chemistry, 66(24), 4514-4518.

Tedeschi, C., Caruso, F., Mohwald, H., \& Kirstein, S. (2000). Adsorption and desorption behavior of an anionic pyrene chromophore in sequentially deposited polyelectrolytedye thin films. Journal of the American Chemical Society, 122(24), 5841-5848.

Vojdani, F., \& Torres, J. A. (1990). Potassium sorbate permeability of methylcellulose and hydroxypropyl methylcellulose coatings - effect of fatty acids. Journal of Food Science, 55(3), 841-846.

Wang, X. Y., Hu, X., Daley, A., Rabotyagova, O., Cebe, P., \& Kaplan, D. L. (2007). Nanolayer biomaterial coatings of silk fibroin for controlled release. Journal of Controlled Release, 121, 190-199.

Wood, K. C., Boedicker, J. O., Lynn, D. M., \& Hammond, P. T. (2005). Tunable drug release from hydrolytically degradable layer-by-layer thin films. Langmuir, 21(4), 1603-1609.

Xia, W., Liu, P., Zhang, J., \& Chen, J. (2011). Biological activities of chitosan and chitooligosaccharides. Food Hydrocolloids, 25(2), 170-179.

Yoo, D., Shiratori, S. S., \& Rubner, M. F. (1998). Controlling bilayer composition and surface wettability of sequentially adsorbed multilayers of weak polyelectrolytes. Macromolecules, 31(13), 4309-4318.

Zhong, Y., Whittington, C. F., Zhang, L., \& Haynie, D. T. (2007). Controlled loading and release of a model drug from polypeptide multilayer nanofilms. Nanomedicine: Nanotechnology, Biology and Medicine, 3(2), 154-160. 